\title{
Análisis comparativo de la inversión-financiación de la innovación entre sectores manufacturero y de servicios en Colombia*
}

\author{
A comparative analysis of investment and funding for \\ innovation in the manufacturing and services industries in \\ Colombia
}

\section{Análise comparativa do invesitimento-financiamento da innovação entre os setores de manufatura e serviços na Colômbia}

\author{
Bernardo Barona-Zuluaga \\ $\mathrm{PhD}$ de la Universidad de Manchester, Reino Unido. Profesor jubilado de la Facultad de Ciencias \\ de la Administración de la Universidad del Valle, Cali - Colombia. \\ bbaronaz@gmail.com
}

\author{
Jorge Alberto Rivera-Godoy \\ Doctor Distinguido "Cum Laude" en Ciencias Económicas y Empresariales, Universidad Autónoma \\ de Madrid, España. Profesor de la Facultad de Ciencias de la Administración de la Universidad del Valle, Cali - Colombia. \\ jorge.rivera@correounivalle.edu.co
}

\section{Resumen}

El artículo tiene como objetivo analizar y comparar cuáles son las actividades de ciencia, tecnología e innovación donde invierten las empresas innovadoras del sector manufacturero y de servicios en Colombia, y cuáles son las fuentes de recursos utilizadas. Para su logro, después de una breve reseña utilizando datos agregados de la inversión en innovación y su financiación en el país, se hace uso de las Encuestas de Desarrollo e Innovación Tecnológica de la Industria Manufacturera [EDIT IV (2007-2008), EDIT VI (2011 - 2012) y de servicios EDITS III (2010-2011)] elaboradas por el Departamento Administrativo Nacional de Estadística - DANE. Para el análisis e interpretación de la información se sigue la teoría económica sobre la financiación empresarial. Los datos de las tres encuestas muestran que a pesar de que existen diferencias entre la inversión y la financiación de la innovación

* Cómo citar: Barona-Zuluaga, B., Rivera-Godoy, J.A. (2017). Análisis comparativo de la inversión-financiación de la innovación entre sectores manufacturero y de servicios en Colombia. Revista Libre Empresa, 14(1), 11-27 http://dx.doi.org/10.18041/libemp.2017.v14n1.27101 
entre los sectores manufacturero y de servicios, estas se centran en la magnitud de la importancia, pero no del escalafón de prioridades para invertir y financiarse: los recursos se usan preferencialmente en la inversión de maquinaria y equipos y la fuente principal de financiación que se emplea son los recursos propios.

\section{Palabras clave}

Finanzas emprendedoras, innovación, financiación de activos intangibles, finanzas corporativas, mezcla de financiación.

\section{Abstract}

This article is aimed at analyzing and comparing scientific, technological, and innovative activities that benefit from investment on the part of innovative companies in the manufacturing and services sectors in Colombia, and identifying the various sources of funding available. To accomplish these objectives, after a brief review based on aggregated data of investment on and funding for innovation in Colombia, DANE [National Statistics Department] Surveys on Technological Development and Innovation in the Manufacturing [EDIT IV (2007-2008), EDIT VI (2011 - 2012) and Services Industries [EDITS III (2010-2011)] were used. The analysis and interpretation of information are based on the economic theory of business funding. The information from the above three surveys reveal that, despite differences between investing and funding for innovation in the manufacturing and services industries, these differences focus on the value reached by the percentages, but not in the ranking of priorities: the resources are used preferentially in the investment of machinery and equipment and the main source of financing that is used is self-funding (equity).

\section{Key words}

Entrepreneurial finance, innovation, funding for intangible assets, corporate finance, funding mix.

\section{Resumo}

$\mathrm{O}$ artigo tem como objetivo analisar e comparar quais são as atividades de ciência, tecnologia e inovação onde investem as empresas inovadoras do setor de fabricação e de serviços na Colômbia e quais são as fontes de recursos usadas. Para sua realização, após uma breve revisão usando dados agregados de investimento em inovação e seu financiamento no país, se faz uso das pesquisas de Desenvolvimento e Inovação Tecnológica da Indústria de Fabricação [EDIT IV (2007-2008), EDIT VI (2011 - 2012) e de serviços EDITS III (2010-2011)] elaboradas pelo Departamento Administrativo Nacional de Estatística (DANE). Para a análise e interpretação da informação, foi seguida a teoria econômica sobre o financiamento empresarial. Os dados das três pesquisas mostram que, embora existam diferenças entre o investimento e o financiamento da inovação entre os setores da manufatura e dos serviços, estas enfocam sobre a magnitude da importância, mas não sobre a escala de prioridades para investir e financiarse: os recursos são usados preferencialmente em investimento em máquinas e equipamentos e a principal fonte de financiamento são utilizados recursos própios. 


\section{Palavras chave}

Finanças empreendedoras, inovação, financiamento de ativos intangíveis, finanças corporativas, mistura de financiamentos.

\section{Introducción}

En Colombia la mayor parte de la inversión en actividades de ciencia, tecnología e innovación - ACTI, es realizada por el sector público. Según cifras del Observatorio Colombiano de Ciencia y Tecnología (2016, p. 23) en el periodo 2005-2015 del total de inversión en innovación de \$36.082.852 millones el sector público invirtió el 56,95\%, el sector privado el $41,15 \%$ y recursos internacionales el 1,90\%.

Los fondos públicos destinados a la financiación de la innovación pueden ser recursos del presupuesto general de la nación y generados mediante el sistema de tratamiento tributario diferenciado. Este último incluye: a) Exenciones: ingresos que se excluyen de la base imponible; b) Deducciones: valores que se permite rebajar de la base imponible; c) Créditos: valores que se permite rebajar de los impuestos; d) Tasas reducidas; e) Diferimientos: postergación o rezago en el pago de un impuesto (Consejo Nacional de Política Económica y Social - CONPES, 2015, p. 3).

Los esfuerzos realizados tanto por el sector público como por el privado en el país, sin embargo, no han sido sistemáticos y los montos invertidos en ACTI han sido insuficientes. Según el CONPES (2016) la inversión en I\&D en el país en el 2015 ascendió al 0,23\% del PIB, porcentaje que es bastante inferior al de países como Brasil, Argentina y México. La inversión promedio durante el periodo 2005-2012 fue de \$1, 1 billones, la cual en un $42 \%$ provino del sector privado. Más recientemente, en el periodo 2013-2015, se produjo un incremento en el valor, cuando subió a \$1, 9 billones, y creció el porcentaje financiado por el sector privado al 47\%; este porcentaje ha sido bastante inferior al obtenido en países desarrollados (en Estados Unidos este porcentaje es del 74\% y en Alemania del 68\%) (CONPES, 2016, p. 69) ${ }^{1}$.

El propósito principal de este artículo es mostrar la clase de inversión en innovación y la forma de financiación que desarrollan las empresas del sector manufacturero y de servicios en Colombia; sus análisis y comparaciones se realizan tomando como principal fuente de información las Encuestas de Desarrollo e Innovación Tecnológica (EDIT IV y EDIT VI para el sector manufacturero y EDITS III para el sector servicios).

Esta investigación se fundamenta en los principios de finanzas corporativas y, dentro de los enfoques que se han desarrollado en los últimos años para explicar la relación innovacióncrecimiento económico, se sigue el de los Sistemas Nacionales de Innovación, que considera que las áreas de focalización y los procesos de innovación en un determinado país son moldeados por "factores institucionales como el sistema científico, el sistema educacional y el mercado laboral, las relaciones industriales y el sector financiero, en su interacción con

1. La debilidad del sistema actual de innovación en Colombia se observa adicionalmente en la reducción significativa del porcentaje de empresas clasificadas como innovadoras en sentido amplio y en sentido estricto (Consejo Privado de Competitividad - CPC, 2015, p. 126). 
las estrategias y estructuras de organizaciones relevantes (en particular de las empresas)" (Hirsch-Kreinsen, 2010, p. 3). En la interpretación que se presenta en este informe de los resultados del análisis de las encuestas del DANE, se hace referencia principalmente a las características de la innovación.

En este estudio se identificaron los activos en los que se vieron reflejadas las actividades de innovaciones empresariales en los periodos cubiertos por la EDIT IV, VI y la EDITS III y se observaron las fuentes de financiación empleadas en el fondeo de dichas inversiones.

\section{Marco teórico}

Para apreciar cómo se financian las actividades de innovación es necesario considerar las características de la innovación. La literatura en la que se analiza la relación inversiónfinanciación de la innovación bajo la perspectiva de la moderna economía financiera ha sido copiosa en las últimas décadas (Ejemplos de esta incluyen a Schumpeter, 1975; Arrow, 1962; O’Sullivan, 2005; Lazonick \& O’Sullivan, 2000; Lazonick (2007); Hirsch-Kreinsen, 2010; Hall \& Lerner, 2010; Arbeláez \& Parra, 2011; Kahn, Melo \& Matos, 2014; Sierra, Malaver \& Vargas, 2009 y Barona-Zuluaga, Rivera-Godoy, Aguilera-Cifuentes \& GarizadoRomán, 2015).

La inversión en innovación presenta características diferentes a las de la inversión en activos convencionales (ver: Schumpeter, 1975 y Arrow, 1962). Esto obedece a que el producto principal de la inversión en investigación y desarrollo (I\&D) es el conocimiento de cómo hacer nuevos bienes y servicios; el uso de este conocimiento por una firma no evita que otra lo use también. En la medida en que el conocimiento no pueda mantenerse en secreto, la firma no podrá apropiarse de los retornos producidos por esta inversión y, por lo tanto, será reacia a invertir, situación que lleva a que haya una sub-provisión de inversión en I\&D en la economía (Hall, 2002, p. 35).

Además, un porcentaje muy elevado de esta inversión está representado en los pagos que se le hacen a personal altamente calificado, como científicos e ingenieros. La naturaleza intangible de estos pagos, aunque conceptualmente crea un activo para la empresa, los hace muy diferentes a un activo físico (Hall, 2002; Hall \& Lerner, 2010). Quien va a financiar esta inversión es consciente de esta naturaleza y de que los activos, además de ser intangibles, no se pueden considerar — como los activos físicos- propiedad de la empresa; la organización puede perder con mayor facilidad estos recursos si las personas la abandonan; además de la intangibilidad, sobre los activos generados con la I\&D no se pueden establecer derechos de propiedad tan claros, como en el caso de los activos tangibles (Rajan \& Zingales, 2003, Ch. 5).

Al contrastar las características de la inversión en activos intangibles con la inversión en activos tangibles, Williamson (1996, p. 185) concluye que los costos de la financiación con deuda son más altos que los de la financiación con patrimonio. Investigaciones recientes de la financiación de la Inversión en I\&D respaldan las conclusiones de la mayor conveniencia de los recursos internos y de las ventajas de la financiación mediante emisión de acciones comparada con la financiación de deuda (Hall \& Lerner, 2010 y Hall, 2002), pero reconocen, 
también, el papel de la deuda (Ayyagari, Demirgüc-Kunt \& Maksimovic, 2011) y el uso de otros mecanismos de financiación como los recursos de capital riesgo (Kortum \& Lerner, 2000) y mediante fondos gubernamentales (Arbeláez \& Parra, 2011).

A continuación se reseñan algunos estudios sobre la financiación de la innovación en Colombia:

Langebaek y Vásquez (2007) analizan los determinantes de la innovación en la industria manufacturera colombiana. Sus resultados muestran que esta actividad evidencia una fuerte relación con el tamaño de la empresa, la presencia de capital extranjero en la propiedad y el nivel de capital humano en la firma.

Sierra, Malaver y Vargas (2009) hacen un estudio sobre la financiación de la innovación en Colombia utilizando, entre otras fuentes de información, las encuestas de innovación aplicadas a la industria manufacturera nacional en 1996 y 2005 (EDIT I y EDIT II) y la Encuesta de Innovación de la Industria de Bogotá y Cundinamarca, en 2005. Entre sus principales resultados está que en las empresas manufactureras predomina la financiación con recursos propios y que este comportamiento es más acentuado en las empresas más grandes y en las de menor tamaño. También encuentran que las empresas más grandes acuden en mayor porcentaje a la banca nacional e internacional. Del mismo modo examinan los mecanismos públicos de financiación y hallan que entre estos el más utilizado es Bancoldex.

Arbeláez y Parra (2011) indican que la inversión en I\&D está afectada negativamente por la propiedad extranjera de la empresa. También estiman que la financiación pública es altamente relevante para la inversión en innovación e I\&D y que los instrumentos públicos son promotores efectivos de la innovación total.

Gómez y Mitchell (2014) hacen un repaso de las actividades de ciencia, tecnología, innovación y generación de emprendimientos dinámicos (CT\&IE) en Colombia y proponen una estrategia para superar el rezago que el país presenta en este campo, en relación con otros de la región. Dicha estrategia se fundamenta en seis pilares, uno de los cuales es "el financiamiento — que puede ser público o privado o una combinación de ambos-, basado en "grants", exenciones tributarias o capital semilla y de riesgo" (p. 1). Se menciona en el trabajo que uno de los principales retos que debe enfrentar el país en el momento es el de incrementar los recursos públicos, especialmente para financiar proyectos de innovación empresarial. También se señala la necesidad de mejorar el proceso de asignación y distribución de recursos para CT\&I del Sistema General de Regalías.

El Consejo Nacional de Política Económica y Social (2015) presenta un nuevo marco conceptual para la política de CT\&I en Colombia basado en el enfoque de sistemas nacionales de innovación y, después de hacer una reseña de la actividad de innovación en el país en los años pasados, plantea una amplia variedad de políticas e instrumentos para estimular la actividad innovadora. 


\section{Metodología}

Se realiza un análisis descriptivo de las muestras de empresas que resultaron después de eliminar de las Encuestas de Desarrollo e Innovación Tecnológica de la Industria Manufacturera (EDIT IV y EDIT VI), y de la Encuesta de Desarrollo e Innovación Tecnológica del Sector Servicios (EDITS III), aquellas empresas clasificadas como no innovadoras porque no obtuvieron innovación, no reportaron tenerla en proceso, o reportaron haber abandonado algún proyecto para la obtención de innovación. Adicionalmente, se excluyeron empresas que no presentaron cifras de inversión/financiación en el periodo estudiado por cada una de las encuestas.

La encuesta, denominada EDIT IV contiene información de 7.683 empresas del sector manufacturero colombiano para el periodo 2007-2008, pero después de la depuración, el número de empresas que conformaron la muestra del presente estudio fue de 2.852 , que representa el $37,10 \%$.

La EDIT VI, realizada para los años 2011-2012, contiene información sobre 9.137 empresas, también del sector manufacturero, pero solo clasificaron para la muestra 1.982 empresas, es decir el 21,69\%.

La EDITS III se aplicó a 5.423 empresas que realizaron actividades de servicios en el período 2010-2011, quedando como muestra del presente estudio 1.835, que constituyen el 33,84\%.

\section{Resultados}

\section{Análisis de los resultados de la EDIT para el sector manufacturero}

\section{Análisis de las actividades de inversión}

Las inversiones en innovación en el periodo 2007-2008 estuvieron concentradas en adquisiciones de maquinaria y equipo $(68,2 \%)$, actividades de $\mathrm{I} \& \mathrm{D}$ internas $(7,6 \%)$, mercadeo de innovaciones $(7,6 \%)$ y asistencia técnica y consultoría (6\%); tecnología de información y telecomunicaciones (5,4\%); mientras que la formación y la capacitación especializada solo alcanzaron un $0,8 \%$ (Tabla 1).

\section{Tabla 1.}

Sector manufactura. Valor invertido en cada una de las actividades científicas, tecnológicas y de innovación. Años 20072008 (Valores monetarios en miles de pesos)

\begin{tabular}{|l|c|r|r|r|r|r|}
\hline & \multicolumn{2}{|c|}{ Año 2007 } & \multicolumn{2}{c|}{ Año 2008 } & \multicolumn{2}{c|}{ Total periodo } \\
\cline { 5 - 7 } & \multicolumn{1}{|c|}{ Valor } & $\%$ & Valor & $\%$ & \multicolumn{2}{c|}{ (2007-2008) } \\
\hline \multicolumn{1}{|c|}{ Tipo de actividad } & 177.419 .413 & 6,2 & 277.923 .514 & 8,8 & 455.342 .927 & 7,6 \\
\hline $\begin{array}{l}\text { Actividades I\&D } \\
\text { Internas }\end{array}$ & 30.194 .624 & 1,1 & 65.114 .464 & 2,1 & 95.309 .088 & 1,6 \\
\hline $\begin{array}{l}\text { Adquisición I\&D } \\
\text { (Externa) }\end{array}$ & & & & & & \\
\hline
\end{tabular}


(Viene de pág.16)

\begin{tabular}{|l|r|r|r|r|r|r|}
\hline $\begin{array}{l}\text { Adquisición } \\
\text { Eaquinaria y }\end{array}$ & 2.032 .445 .989 & 71,2 & 2.072 .049 .852 & 65,5 & 4.104 .495 .841 & 68,2 \\
\hline $\begin{array}{l}\text { Tecnologías de } \\
\text { Información y } \\
\text { Telecomunicaciones }\end{array}$ & 153.978 .532 & 5,4 & 173.784 .466 & 5,5 & 327.762 .998 & 5,4 \\
\hline $\begin{array}{l}\text { Mercadeo de } \\
\text { Innovaciones }\end{array}$ & 207.062 .888 & 7,3 & 252.821 .584 & 8 & 459.884 .472 & 7,6 \\
\hline $\begin{array}{l}\text { Transferencia de } \\
\text { Tecnología }\end{array}$ & 26.130 .229 & 0,9 & 42.375 .283 & 1,3 & 68.505 .512 & 1,1 \\
\hline $\begin{array}{l}\text { Asistencia Técnica } \\
\text { y Consultoría }\end{array}$ & 161.241 .601 & 5,6 & 201.525 .349 & 6,4 & 362.766 .950 & 6,0 \\
\hline $\begin{array}{l}\text { Ingeniería y Diseño } \\
\text { Industrial }\end{array}$ & 44.104 .286 & 1,5 & 48.828 .092 & 1,5 & 92.932 .378 & 1,5 \\
\hline $\begin{array}{l}\text { Formación y } \\
\text { Capacitación } \\
\text { Especializada }\end{array}$ & 21.871 .923 & 0,8 & 26.880 .934 & 0,9 & 48.752 .857 & 0,8 \\
\hline Total & 2.854 .449 .485 & 100,0 & 3.161 .303 .538 & 100,0 & 6.015 .753 .023 & 100,0 \\
\hline
\end{tabular}

Fuente: Barona-Zuluaga et al. (2015, p. 85)

En los años 2011 y 2012 la inversión en maquinaria y equipo disminuyó con respecto al periodo 2007-2008, alcanzando participaciones del $52,54 \%$ y $54,44 \%$, respectivamente, y cediendo terreno a actividades de I\&D internas (16,45\% y $15,77 \%)$, mercadeo de innovaciones $(9,79 \%$ y 9,85\%) e inversión en tecnologías de la información y las comunicaciones $(10,49 \%$ y $7,97 \%)$. Mientras que las actividades de formación y capacitación continuaron siendo poco importantes en los años 2011 y 2012, con porcentajes inferiores al 1\% (Tabla 2).

\section{Tabla 2.}

Sector manufactura. Valor invertido en cada una de las actividades científicas, tecnológicas y de innovación. Años 20112012 (Valores monetarios en miles de pesos)

\begin{tabular}{|l|c|c|c|c|c|c|}
\hline & \multicolumn{2}{|c|}{ Año 2011 } & \multicolumn{2}{c|}{ Año 2012 } & \multicolumn{2}{c|}{$\begin{array}{c}\text { Total periodo } \\
\text { (2011-2012) }\end{array}$} \\
\hline \multicolumn{1}{|c|}{ Tipo de actividad } & Valor & $\%$ & Valor & $\%$ & Valor & $\%$ \\
\hline $\begin{array}{l}\text { Actividades I\&D } \\
\text { Internas }\end{array}$ & 305.875 .427 & 16,45 & 382.132 .257 & 15,77 & 688.007 .684 & 15,77 \\
\hline $\begin{array}{l}\text { Adquisición I\&D } \\
\text { Externa }\end{array}$ & 21.438 .317 & 1,15 & 35.735 .713 & 1,43 & 57.174 .030 & 1,32 \\
\hline $\begin{array}{l}\text { Adquisición } \\
\text { Maquinaria y } \\
\text { Equipo }\end{array}$ & 976.411 .663 & 52,54 & 1.362 .097 .423 & 54,44 & 2.338 .509 .086 & 53,62 \\
\hline
\end{tabular}

(Continúa en pág. 18) 
(Viene de pág. 18)

\begin{tabular}{|l|r|r|r|r|r|r|}
\hline $\begin{array}{l}\text { Tecnologías de } \\
\text { Información y } \\
\text { Telecomunicaciones }\end{array}$ & 195.010 .339 & 10,49 & 199.512 .521 & 7,97 & 394.522 .860 & 9,05 \\
\hline $\begin{array}{l}\text { Mercadeo de } \\
\text { Innovaciones }\end{array}$ & 181.998 .462 & 9,79 & 247.433 .754 & 9,85 & 429.432 .216 & 9,85 \\
\hline $\begin{array}{l}\text { Transferencia de } \\
\text { Tecnología }\end{array}$ & 49.841 .894 & 2,68 & 90.651 .532 & 3,62 & 140.493 .426 & 3,23 \\
\hline $\begin{array}{l}\text { Asistencia Técnica y } \\
\text { Consultoría }\end{array}$ & 85.391 .575 & 4,59 & 106.453 .304 & 4,25 & 191.844 .879 & 4,39 \\
\hline $\begin{array}{l}\text { Ingeniería y Diseño } \\
\text { Industrial }\end{array}$ & 29.705 .887 & 1,6 & 63.592 .372 & 2,54 & 93.298 .259 & 2,14 \\
\hline $\begin{array}{l}\text { Formación y } \\
\text { Capacitación } \\
\text { Especializada }\end{array}$ & 12.894 .223 & 0,69 & 14.391 .925 & 0,58 & 27.286 .148 & 0,63 \\
\hline Total & 1.858 .567 .787 & 100,0 & 2.502 .000 .801 & 100,0 & 4.360 .568 .588 & 100,0 \\
\hline
\end{tabular}

Fuente: Cálculos de los autores a partir de la información de la EDIT VI facilitada por el DANE.

\section{Análisis de la financiación}

Los recursos propios contribuyeron en el periodo 2007-2008 con el 76,3\% de la financiación, en tanto que los recursos de la banca privada lo hicieron con el 18,2\%, como se muestra en la Tabla 3.

\section{Tabla 3.}

Monto e importancia relativa de las diferentes fuentes de financiación utilizadas por las empresas. Año 2007-2008

(Valores monetarios en miles de pesos)

\begin{tabular}{|l|r|r|}
\hline \multicolumn{1}{|c|}{ Tipo de financiación } & \multicolumn{1}{c|}{ Valor } & \multicolumn{1}{c|}{$\%$} \\
\hline Recursos propios de la empresa & 4.592 .862 .599 & 76,3 \\
\hline Recursos propios otras empresas del grupo & 95.212 .583 & 1,6 \\
\hline Recursos públicos & 43.328 .451 & 0,7 \\
\hline Recursos banca privada nacional & 1.093 .262 .396 & 18,2 \\
\hline Recursos banca privada extranjera & 142.603 .283 & 0,4 \\
\hline Recursos de otras empresas nacional & 11.184 .959 & 0,2 \\
\hline Recursos de otras empresas extranjero & 0 & 0,1 \\
\hline Recursos de capital nacional & 5.086 .020 & 0,5 \\
\hline Recursos de capital extranjero & 28.891 .059 & 0,1 \\
\hline Recursos cooperación o donaciones nacional & 3.071 .608 & 0 \\
\hline Recursos cooperación o donaciones extranjero & 250.165 & 100,0 \\
\hline Total & 6.015 .753 .123 & \\
\hline
\end{tabular}

Fuente: Barona-Zuluaga et al. (2015, p. 85). 
La financiación bancaria se ve incrementada, si se considera el 2,4\% con la que participa la banca privada extranjera. Con contribuciones bajas aparecen los recursos públicos (0,7\%), y los recursos de capitales nacionales y extranjeros (0,1\% y 0,5\%, respectivamente).

En la Tabla 4 se resumen las fuentes de financiación empleadas en los años 2011 y 2012, así como su importancia dentro del total de fuentes de financiación.

\section{Tabla 4.}

Sector manufactura. Monto e importancia relativa de las diferentes fuentes de financiación utilizadas por las empresas. Años 2011-2012 (Valores monetarios en miles de pesos)

\begin{tabular}{|l|r|r|r|r|}
\hline \multicolumn{1}{|c|}{ Tipo de financiación } & \multicolumn{1}{c|}{2011} & \multicolumn{2}{c|}{2012} \\
\hline Recursos propios de la empresa & 1.432 .195 .567 & 77,06 & 1.877 .594 .613 & 75,04 \\
\hline $\begin{array}{l}\text { Recursos propios otras empresas del } \\
\text { grupo }\end{array}$ & 28.146 .540 & 1,51 & 36.613 .640 & 1,46 \\
\hline Recursos públicos & 8.495 .447 & 0,46 & 26.982 .877 & 1,08 \\
\hline Recursos banca privada nacional & 358.888 .148 & 19,31 & 488.922 .116 & 19,54 \\
\hline Recursos banca privada extranjero & 14.068 .862 & 0,76 & 34.393 .154 & 1,38 \\
\hline Recursos de otras empresas nacional & 4.983 .007 & 0,27 & 1.853 .917 & 0,07 \\
\hline Recursos de otras empresas extranjero & & 0 & 30.764 .437 & 1,23 \\
\hline Recursos de capital nacional & 7.810 .266 & 0,42 & 2.133 .811 & 0,09 \\
\hline Recursos de capital extranjero & & 0 & 458.355 & 0,02 \\
\hline $\begin{array}{l}\text { Recursos cooperación o donaciones } \\
\text { nacional }\end{array}$ & 2.625 .617 & 0,14 & 1.568 .780 & 0,06 \\
\hline $\begin{array}{l}\text { Recursos cooperación o donaciones } \\
\text { extranjero }\end{array}$ & 1.354 .333 & 0,07 & 715.101 & 0,03 \\
\hline Total & 1.858 .567 .787 & 100 & 2.502 .000 .801 & 100,0 \\
\hline
\end{tabular}

Fuente: Cálculos de los autores a partir de la información de la EDIT VI facilitada por el DANE.

$\mathrm{Al}$ analizar las cifras de la Tabla 4 se observa que los recursos propios conservaron su mayor participación en el 2011 (77,06\%) y en el 2012 (75,04\%). Mientras tanto los préstamos de la banca privada nacional aumentaron a 19,31\% en el 2011 y a 19,54\% en el 2012; pero la banca privada extranjera disminuyó su participación al 0,76\% en el 2011 y se vuelve a recuperar un poco en el 2012, al pasar al 1,38\%. La importancia de los recursos públicos ${ }^{2}$ se redujo al 0,46\% en el 2011 y se recuperó algo en el 2012 al pasar al 1,08\%. Se debe resaltar

2. El financiamiento con recursos públicos proviene principalmente de las líneas de cofinanciación de Fomipyme, SENA y Colciencias. Las líneas de crédito durante los periodos estudiados fueron las de Bancoldex y Bancoldex-Colciencias. Otros recursos públicos, por montos generalmente inferiores a los de las fuentes anteriores, proceden de fondos departamentales o municipales de ciencia y tecnología. 
lo poco representativos que fueron los recursos de capital privado, tanto nacional como extranjero, con un porcentaje inferior al 0,5\% en ambos años.

Al examinar las fuentes de financiación externas distintas de financiación con recursos propios, se observa que la banca privada nacional ha sido la fuente externa más importante en los tres períodos, con un porcentaje igual o superior al 75,9\%; su importancia creció en 2011 con un $84,2 \%$, pero se redujo a 78,3\% en el 2012 (Tabla 5).

\section{Tabla 5.}

Sector manufactura. Fuentes de financiación diferentes de recursos internos, empleadas por las empresas de la muestra comprendidas en la EDIT IV y la EDIT VI.

\begin{tabular}{|l|c|c|c|}
\hline & $\mathbf{2 0 1 2}$ & $\mathbf{2 0 1 1}$ & $\mathbf{2 0 0 7 - 2 0 0 8}$ \\
\hline Tipo de financiación & $\%$ & $\%$ & $\%$ \\
\hline Recursos propios otras empresas del grupo & 5,9 & 6,6 & 3,2 \\
\hline Recursos públicos & 4,3 & 2,0 & 7,5 \\
\hline Recursos banca privada nacional & 78,3 & 84,2 & 75,9 \\
\hline Recursos banca privada extranjero & 5,5 & 3,3 & 3,2 \\
\hline Recursos de otras empresas nacional & 0,3 & 1,2 & 2,7 \\
\hline Recursos de otras empresas extranjero & 4,9 & 0,0 & 0,0 \\
\hline Recursos de capital nacional & 0,3 & 1,8 & 5,2 \\
\hline Recursos de capital extranjero & 0,1 & 0,0 & 0,3 \\
\hline Recursos cooperación o donaciones nacional & 0,3 & 0,6 & 1,5 \\
\hline Recursos cooperación o donaciones extranjero & 0,1 & 0,3 & 0,6 \\
\hline Total & 100,0 & 100,0 & 100,0 \\
\hline
\end{tabular}

Fuente: Cálculos de los autores con base en información EDIT VI y Barona et al. (2015, p. 87)

Además, cabe destacar la gran reducción del porcentaje de recursos públicos entre 20072008 y el 2011 (7,5\% a 2,0\%), con una recuperación en el 2012 (4,3\%), pero inferior al alcanzado en los años 2007-2008.

En cuanto a la participación de recursos de capital privado nacional en el período 20072008, esta fue de 5,20\%; porcentaje bajo, que se redujo aún más en los años 2011 (1,8\%) y $2012(0,3 \%)$. En relación con los recursos de capital extranjero se observa que en estos tres períodos no alcanzan a superar el 0,3\%, no obstante el empeño del gobierno por atraer fondos extranjeros (Barona \& Rivera, 2012). Otro hecho que se debe resaltar es que los recursos de otras empresas del extranjero presentaron un incremento considerable, al pasar de 0\% en el 2007-2008, igual en el 2011, a 4,9\% en el 2012.

Al comparar las inversiones en innovación y sus fuentes de financiación empleadas, entre el periodo 2007-2008 y los años 2011 y 2012 se evidencia: 
1. El porcentaje de inversiones en activos intangibles aumentó y el de los activos tangibles disminuyó.

2. Como los activos intangibles son más difíciles de financiar por la banca comercial, se esperaría que este cambio representara una mayor presión sobre los recursos internos de las empresas. Los datos de la EDIT VI indican que, efectivamente, los recursos propios han continuado financiando la mayor parte de las inversiones en innovación.

3. Igualmente, el cambio en la composición de las inversiones haría pensar que la importancia de las fuentes recursos públicos y fondos de capital nacionales y extranjeros debería aumentar ${ }^{3}$; sin embargo, estos recursos no han respondido en forma adecuada a los cambios en la composición de la inversión, presentándose una disminución en su importancia relativa.

4. Los recursos provistos por la banca nacional mostraron un ligero crecimiento en su importancia relativa, aunque sigue siendo inferior al $20 \%$.

\section{Análisis de los resultados de la EDITS para el sector servicios}

\section{La inversión en innovación en las empresas del sector.}

Como se observa en la Tabla 6, la mayor inversión en ACTI en el período 2010-2011 fue en la adquisición de maquinaria y equipo, con un 33,5\%, mostrando preponderancia en ambos años, aunque con una reducción en el último año (2010: 42,24\% y 2011: $26,34 \%$ ). La segunda inversión más significativa fue en tecnologías de la información y las comunicaciones con el 22,25\% (2010: 21,06\% y 2011: 23,24\%). El tercer lugar en importancia lo alcanzó la inversión en actividades de investigación y desarrollo internas con un 14,34\%, seguido muy de cerca por otras actividades con un 14,24\%. Estas dos últimas aumentaron su participación en el año 2011, en contraste con la reducción de la adquisición de maquinaria y equipo.

\section{Tabla 6.}

Sector servicios. Valor invertido en cada una de las actividades científicas, tecnológicas y de innovación. Años 2010-2011 (Valores monetarios en miles de pesos)

\begin{tabular}{|l|c|c|c|c|c|c|}
\hline & \multicolumn{2}{|c|}{ Año 2010 } & \multicolumn{2}{c|}{ Año 2011 } & \multicolumn{2}{c|}{$\begin{array}{c}\text { Total periodo } \\
\text { (2010-2011) }\end{array}$} \\
\hline Tipo de actividad & Valor & $\%$ & Valor & $\%$ & Valor & $\%$ \\
\hline $\begin{array}{l}\text { Actividades I\&D } \\
\text { Internas }\end{array}$ & 336.462 .678 & 13,57 & 448.977 .054 & 15,00 & 785.439 .732 & 14,34 \\
\hline $\begin{array}{l}\text { Adquisición I\&D } \\
\text { Externa }\end{array}$ & 87.456 .906 & 3,53 & 147.257 .222 & 4,92 & 234.714 .128 & 4,29 \\
\hline $\begin{array}{l}\text { Adquisición } \\
\text { Maquinaria y } \\
\text { Equipo }\end{array}$ & 1.047 .710 .844 & 42,24 & 788.777 .537 & 26,34 & 1.836 .488 .381 & 33,55 \\
\hline
\end{tabular}

3. En varios estudios se indica la importancia que puede tener la disponibilidad de estas fuentes de recursos para impulsar la innovación que toma forma de activos intangibles (Ejes.: Kahn, Melo y Matos, 2014; Kerr y Nanda, 2014). 
(Viene de pág. 21)

\begin{tabular}{|l|r|r|r|r|r|c|}
\hline $\begin{array}{l}\text { Tecnologías de } \\
\text { Información y } \\
\text { Comunicaciones }\end{array}$ & 522.258 .694 & 21,06 & 695.906 .594 & 23,24 & 1.218 .165 .288 & 22,25 \\
\hline $\begin{array}{l}\text { Mercadeo de } \\
\text { Innovaciones }\end{array}$ & 179.539 .242 & 7,24 & 262.631 .815 & 8,77 & 442.171 .057 & 8,07 \\
\hline $\begin{array}{l}\text { Transferencia de } \\
\text { Tecnología }\end{array}$ & 17.826 .971 & 0,72 & 64.299 .521 & 2,15 & 82.126 .492 & 1,50 \\
\hline $\begin{array}{l}\text { Formación y } \\
\text { Capacitación } \\
\text { Especializada }\end{array}$ & 41.912 .129 & 1,69 & 53.689 .221 & 1,79 & 95.601 .350 & 1,75 \\
\hline Otras actividades & 247.071 .449 & 9,96 & 532.562 .542 & 17,79 & 779.633 .991 & 14,24 \\
\hline Total & 2.480 .238 .913 & 100,0 & 2.994 .101 .506 & 100,0 & 5.474 .340 .419 & 100,0 \\
\hline
\end{tabular}

a/ Asistencia técnica y consultoría e ingeniería y diseño industrial.

Fuente: Cálculos de los autores a partir de la información de la EDITS III facilitada por el DANE.

\section{Análisis de la financiación}

En la Tabla 7 se observa que los recursos propios de la empresa son, de lejos, la más importante fuente de financiación en las actividades de innovación con un $62,14 \%$ en el 2010 y un $68,46 \%$ en el 2011; seguida por la banca privada nacional con el $17,45 \%$ en el 2010 y $19,41 \%$ en el 2011; y luego por la banca privada extranjera con un $10,59 \%$ en el 2010, pero viniéndose a menos en el 2011 con tan solo un $0,16 \%$. Los recursos públicos alcanzaron el 2,7\% en el 2010 y el 4,17\% en el 2011, y los recursos de capital nacional con $3,65 \%$ en el 2010 y 3,47\% en el 2011; mientras que las demás fuentes de recursos no superaron el 1,6\%.

\section{Tabla 7.}

Sector servicios. Fuentes de recursos empleados en financiación de actividades de innovación.

Año 2010-2011 (Valores monetarios en miles de pesos)

\begin{tabular}{|l|r|r|r|r|}
\hline \multicolumn{1}{|c|}{ Tipo de financiación } & \multicolumn{2}{c|}{$\mathbf{2 0 1 0}$} & \multicolumn{2}{c|}{2011} \\
\hline Recursos propios de la empresa & \multicolumn{1}{c|}{ Valor } & \multicolumn{1}{c|}{$\%$} & \multicolumn{1}{c|}{ Valor } & \multicolumn{1}{c|}{$\%$} \\
\hline $\begin{array}{l}\text { Recursos propios otras empresas del } \\
\text { grupo }\end{array}$ & 1.541 .131 .999 & 62,14 & 2.049 .798 .814 & 68,46 \\
\hline Recursos públicos & 67.238 .707 & 1,46 & 48.049 .680 & 1,60 \\
\hline Recursos banca privada nacional & 432.737 .304 & 17,45 & 581.113 .477 & 19,41 \\
\hline Recursos banca privada extranjera & 262.618 .497 & 10,59 & 4.892 .296 & 0,16 \\
\hline Recursos de otras empresas nacional & 10.326 .697 & 0,42 & 16.706 .418 & 0,56 \\
\hline Recursos de otras empresas extranjera & 1.524 .886 & 0,06 & 1.530 .391 & 0,05 \\
\hline
\end{tabular}

(Continúa en pág. 23) 
(Viene de pág. 22)

\begin{tabular}{|l|r|r|r|r|}
\hline Recursos de capital nacional & 90.501 .288 & 3,65 & 103.749 .612 & 3,47 \\
\hline Recursos de capital extranjero & 1.993 .442 & 0,08 & 1.377 .698 & 0,05 \\
\hline $\begin{array}{l}\text { Recursos cooperación o donaciones } \\
\text { nacional }\end{array}$ & 27.171 .813 & 1,10 & 47.169 .313 & 1,58 \\
\hline $\begin{array}{l}\text { Recursos cooperación o donaciones } \\
\text { extranjero }\end{array}$ & 8.916 .222 & 0,36 & 14.735 .302 & 0,49 \\
\hline Total & 2.480 .238 .913 & 100 & 2.994 .101 .506 & 100,0 \\
\hline
\end{tabular}

Fuente: Cálculos de los autores a partir de la información de la EDITS III facilitada por el DANE.

En la información suministrada por el DANE sobre los resultados de las encuestas EDIT y EDITS no es posible identificar el grado en que las empresas al invertir recursos propios en la financiación de la innovación han utilizado las deducciones tributarias, establecidas por primera vez en Colombia mediante la Ley 6 de 1992 (CONPES, 2015, p. 11). La ley vigente establece que las personas que realicen inversiones en proyectos calificados por el Consejo Nacional de Beneficios Tributarios en Ciencia y Tecnología e Innovación (CNBT) como de investigación, desarrollo tecnológico o innovación, de acuerdo con los criterios y las condiciones definidos por el Consejo Nacional de Política Económica y Social mediante un documento CONPES, podrán acceder a un cupo de reducción de renta del $100 \%$ de la inversión realizada y podrán descontar de su impuesto sobre la renta a cargo el $25 \%$ del valor de lo invertido en dichos proyectos en el período gravable en que se realizó la inversión, acorde con lo estipulado en los Artículos 158-1 y 256 y 258 del Estatuto Tributario 2017. Aunque los beneficios tributarios han jugado un papel importante en la financiación de la innovación en el país, según el CPC, el beneficio real neto de las deducciones de renta a inversiones de CT\&I pasó de 28,3\% del monto de la inversión al 8,3\% entre el 2012 y el 2015. Según el análisis hecho por el CPC, varios factores explican esta deducción: en primer lugar está la reducción del impuesto de renta, y la creación del CREE mediante la Ley 1607 del 2012 y posteriormente la sobretasa del CREE mediante la Ley 1739 de 2014; en segundo lugar, la Ley 450 de 2011 que determinó que el beneficio solo se podía otorgar a proyectos que se realizaran a través de centros o grupos de investigación reconocidos por Colciencias, y estableció que el beneficio solamente aplica a inversiones en proyectos de I\&D y no a inversiones en Innovación (CPC, 2015, p. 229).

El documento CONPES 3834 de 2015 hace un diagnóstico del proceso de implementación de los beneficios tributarios de CT\&I en Colombia llegando a la conclusión de que existen dos problemas centrales: el primero hace referencia al alcance limitado del incentivo de deducción para estimular la inversión privada en ACTI y el segundo las dificultades relacionadas con el uso del instrumento (p.15).

Entre las limitaciones de alcance están: el que los usuarios del instrumento se concentren en grandes empresas y en pocos sectores, dejando por fuera las pymes que no declaran renta líquida, así como sectores productivos que realizan I+D+i en menor escala. 
Dice el CONPES (2015, p. 18) que el beneficio efectivo de la deducción por una inversión en ciencia, tecnología e innovación puede resultar insuficiente para compensar el riesgo asociado a este tipo de proyectos: los cálculos realizados muestran que el beneficio efectivo sería del 22,3\% de la inversión inicial (CONPES, 2015, p. 19). Este mismo documento evidencia que, de los recursos totales empleados en la financiación mediante este mecanismo de deducciones, el porcentaje más alto fue para grandes empresas $(67 \%$ en $2012 ; 23 \%$ en 2013 y 74\% en 2014) (p. 20). Se nota que el 79\% de los proyectos presentados por empresas grandes fueron aprobados (p. 25). El documento hace notar una gran concentración, no solo en la asignación de recursos, sino también en la presentación de proyectos, lo cual se ejemplifica con un caso de una sola empresa beneficiaria que obtuvo cerca de 383 proyectos aprobados en los 13 años más recientes (p. 25). En cuanto a limitaciones para el uso del instrumento, el mencionado documento CONPES señala, entre otras, un bajo conocimiento del instrumento por parte de beneficiarios potenciales y los bajos niveles de aprobación de los proyectos presentados (Ver anexo A del documento CONPES 3834). Según este documento, durante el periodo 2001-2014 la tasa de aprobación fue del 72\%, presentándose en los años 2012 y 2013 las tasas más bajas de aprobación: 52\% y 51\%, respectivamente (p. 23).

\section{Conclusiones}

En este estudio se evidencia que la mayor parte de la inversión en innovación en Colombia, adelantada por empresas del sector privado, según la encuesta EDIT, se concentró en activos tangibles. En el sector manufacturero los resultados de la EDIT IV y la EDIT VI muestran que la adquisición de maquinaria representó el 68,2\% en el período 2007-2008 y el 53,62\% en el período 2011-2012; en el sector servicios (EDITS III) este porcentaje fue también el más importante, aunque bastante inferior en relación con el manufacturero, representando solo el 33,55\% en el período 2010-2011. La inversión en intangibles como las tecnologías de la información y las comunicaciones en el sector manufacturero en el período 2007-2008 fue de 5,4\% y en el período 2011-2012 de 9,05\%, inferior al sector servicios con promedio de 22,25\% entre el 2010-2011. Lo mismo sucede con la asistencia técnica y consultoría e ingeniería y diseño industrial que representó para el sector manufacturero el 7,5\% en el período 2007-2008, y 6,53\% en el período 2011-2012, en tanto que para el sector servicios fue de 14,24\% en el período 2010-2011. Esta mayor inversión en intangibles en el sector servicios también se corrobora, aunque con porcentajes más pequeños, cuando se compara la inversión en formación y capacitación especializada, que fue de 1,75\% (2010-2011), versus las registradas en el sector manufacturero: 0,8\% (2007-2008) y 0,63 (2011-2012).

En cuanto a la financiación, se anota que la principal fuente de fondos empleada fue el recurso propio; evidencia que se ajusta a la teoría desarrollada, entre otros, por economistas como Williamson (1988) y Hall y Lerner (2010) que, teniendo principalmente como referencia las condiciones de los mercados financieros de Estados Unidos, sugieren que la fuente de financiación más idónea para financiar innovación son los recursos internos. Estudios adelantados por otros investigadores muestran que el principal instrumento utilizado por el Gobierno nacional para estimular la inversión de recursos propios en innovación por parte de las empresas, y que son las deducciones tributarias, no han logrado plenamente los 
objetivos buscados debido al alcance limitado de las deducciones permitidas para estimular la inversión privada en ACTI y por las dificultades relacionadas con el uso del instrumento, mencionadas anteriormente.

De otra parte, algunos economistas han basado sus análisis en economías como la alemana, en la que los bancos han tenido un papel fundamental en la financiación de la industria en general y de la actividad innovadora en particular (O'Sullivan, 2005; Lazonick, 2007; Hirsch-Kreinsen, 2010), y han señalado algunas bondades que tiene la financiación bancaria para financiar este tipo de actividades, anotando ciertas deficiencias que tienen los mercados accionarios para este mismo propósito.

Resultados de investigaciones en los que se analizan datos de varios países confirman el papel dominante de los recursos internos, pero se encuentra también, como en el estudio reportado aquí, que la banca ha participado de manera destacada en la financiación de la innovación. Así, por ejemplo, Ayyagari et al. (2011) analizan las prácticas de innovación de 19.000 firmas pymes en 47 economías en desarrollo; sus resultados muestran que existe una relación positiva significativa entre el uso de financiación externa (definida como todas las fuentes de financiación con excepción de los recursos propios) y el grado de innovación. Adicionalmente anotan estos autores que la financiación bancaria resulta asociada de manera positiva con el mejoramiento de las líneas de productos existentes, la apertura de una nueva planta y la firma de aventuras conjuntas con socios extranjeros. Barona-Zuluaga et al. (2015), utilizando los resultados de la EDIT IV, encuentran que los recursos propios y los provistos por el sistema bancario son los que han sido más intensamente utilizados por las empresas innovadoras colombianas. Sierra, Malaver y Vargas (2009), empleando datos de la Encuesta de Innovación y Desarrollo Tecnológico en Bogotá y Cundinamarca, realizada en el 2005, evidencian también la preponderancia de los recursos internos en la financiación de la innovación ${ }^{4}$.

Con el fin de validar algunas de los hallazgos del estudio presente sobre la relación tipo de inversión realizada (en activos tangibles o intangibles) y fuente de financiación empleada, es conveniente en futuras investigaciones analizar la información a un nivel de desagregación mayor que el empleado aquí, dada la heterogeneidad de las empresas agrupadas en las dos grandes categorías manufactura y servicios ${ }^{5}$. En investigaciones futuras se debería también poder hacer análisis de las inversiones realizadas y las fuentes de financiación empleadas categorizando las empresas de acuerdo con su edad; en el presente estudio esto no se hizo pues el DANE no accedió a facilitar a los investigadores información que permitiera identificar la edad de cada una de las empresas que conformaron las muestras en las encuestas EDIT IV y VI y EDITS III.

4 Al respecto se preguntan si los recursos internos han sido suficientes para financiar la actividad innovadora 0 si " iserá más bien que, dadas las restricciones financieras externas (desconocimiento de las fuentes oficiales, inconveniencia o exageradas condiciones de las fuentes privadas, inexistencia de capital de riesgo), las empresas se resignan a hacer lo que pueden con los fondos disponibles?" (p. 206).

5 Es de esperarse, por ejemplo, que la naturaleza de las inversiones hechas por las empresas de la Sección D que pertenecen a la división 15, Elaboración de productos alimenticios y bebidas, sea diferente a la de las empresas que pertenecen a la División 27, Fabricación de productos metalúrgicos básicos (clasificaciones hacen referencia a la CIIU, Revisión 3 adaptada para Colombia). En este caso, la consolidación de todas las empresas en la categoría de industria manufacturera dificulta la identificación precisa de las relaciones que el estudio trató de identificar. 


\section{Conflicto de intereses}

Los autores declaran no tener ningún conflicto de intereses.

\section{Referencias bibliográficas}

1. Arbeláez, M. \& Parra, M. (2011). Innovation, R\&D investment and productivity in Colombian Firms. IDB. Working Paper Series No. IDB-WP-251.

2. Arrow, K. (1962). Economic welfare and the allocation of recourses for invention. The rate and direction on inventive activity. Princeton, NJ: Princeton University Press.

3. Ayyagari, M., Demirgüc-Kunt, A. \& Maksimovic, V. (2011). Firm innovation in emerging markets: The role of finance, governance and competition. Journal of Financial and Quantitative Analysis, 46, 1545-1580.

4. Barona, B. \& Rivera, J. (2012). Finanzas emprendedoras en Colombia. Conceptos y resultados de algunos estudios. Cali, Colombia: Facultad de Ciencias de la Administración, Universidad del Valle.

5. Barona-Zuluaga, B., Rivera-Godoy, J., Aguilera-Cifuentes, C. \& Garizado-Román, P. (2015). Financiación de la innovación en Colombia. Entramado, 11(1), 80-93.

6. Consejo Nacional de Política Económica y Social - CONPES (2015). Documento CONPES 3834. Lineamientos de política para estimular la inversión privada en ciencia, tecnología e innovación a través de deducciones tributarias. República de Colombia: Departamento Nacional de Planeación.

7. Consejo Nacional de Política Económica y Social - CONPES (2016). Documento CONPES. Política de Ciencia, Tecnología e Innovación, 2016-2025. Versión para discusión: 22/06/2016. República de Colombia: Departamento Nacional de Planeación.

8. Consejo Privado de Competitividad - CPC (2015). Informe Nacional de Competitividad 2015-2016. Recuperado el 15 de junio de 2016 de http://compite.com.co/wp-content/ uploads/2016/05/INC-2015-2016.pdf

9. Estatuto tributario 2017. Legis Editores S.A.

10. Gómez, H. \& Mitchell, D. (2014). Innovación y emprendimiento en Colombia: balance, perspectivas y recomendaciones de política 2014-2018. Cuadernos de Fedesarrollo, 50.

11. Hall, B. (2002). The financing of research and development. Oxford Review of Economic Policy, 18(1), 35-51.

12. Hall, B. \& Lerner, J. (2010). The financing of R\&D and innovation. En: B. Hall \& N. Rosenberg (eds.), Handbook in Economics (chap.14). Amsterdam: Elsevier.

13. Hirsch-Kreinsen, H. (2010). Financial Market and Innovation. Paper presented at the summer Conference 2010 on Opening up Innovation: Strategy, Organization and Technology at Imperial College London Business School, June 16-18.

14. Kahn, M., Melo, L. \& Matos, M. (eds.) (2014). Financing innovation. London: Routledge.

15. Kerr, W. \& Nanda, R. (2014). Financing innovation. Harvard Business Scholl Working Paper 15-034.

16. Kortum, S. \& Lerner, J. (2000). Assessing the contribution of venture capital to innovation. RAND Journal of Economics, 31(4), 674-692.

17. Langebaek, A. \& Vásquez, D. (2007). Determinantes de la actividad innovadora en la industria manufacturera colombiana. Banco de la República. Working Paper No. 433. 
18. Lazonick, W. (2007). The US stock market and the governance of innovative enterprise. Industrial and Corporate Change, 16(6), 983-1035.

19. Lazonick, W. \& O’Sullivan, M. (2000). Maximizing shareholder value: a new ideology for corporate governance. Economy and Society, 29(1), 13-35.

20. O’Sullivan, M. (2005) Finance and Innovation. En: Fagerberg, J., Mowery. D. \& Nelson, R. (eds.). The Oxford handbook of innovation. Oxford: Oxford University Press.

21. Observatorio Colombiano de Ciencia y Tecnología - OCyT (2016). Indicadores de Ciencia y Tecnología en Colombia 2015. Bogotá D.C. Recuperado en noviembre 7 de 2016 de http://ocyt.org.co/Portals/0/LibrosPDF/OCyT\%20Indicadores\%202015.pdf

22. Rajan, R. \& Zingales, L. (2003). Saving capitalism from the capitalists. New York, USA: Crown Business.

23. Schumpeter, J. A. (1975). Capitalism, socialism and democracy. NY: Harper Perennial. (Reimpresión de la $3^{\mathrm{a}}$ edición de 1950; primera edición publicada en 1942).

24. Sierra, J., Malaver, F. \& Vargas, M. (2009). La financiación de la innovación: un análisis a partir de la encuesta de innovación de Bogotá y Cundinamarca. En: J. Robledo, F. Malaver \& M. Vargas (eds.), Encuestas, datos y descubrimiento de conocimiento sobre la innovación en Colombia (pp. 175-210). Bogotá: Fundación Cultural Javeriana de Artes Gráficas.

25. Williamson, O. (1988). Corporate finance and corporate governance. Journal of Finance 43, 567-591.

26. Williamson, O. (1996). The mechanisms of governance. Oxford, UK: Oxford University Press. 